\title{
The Importance of Bronchial Epithelial Junction Integrity in Asthma
}

\section{Hongbing Xiao ${ }^{1}$, Alicia N Rizzo², Jessica Siegler ${ }^{2}$ and Weiguo Chen ${ }^{2 *}$}

'Department of General Surgery, Shanghai Tenth People's Hospital, Tongji University, China

${ }^{2}$ Section of Pulmonary, Critical Care, Sleep and Allergy, Institute for Personalized Respiratory Medicine, University of Illinois at Chicago, USA

\begin{abstract}
Bronchial epithelial junctions provide not only a physical barrier, but also an immune barrier against the allergens of asthma. Epithelial junction integrity is closely related to the severity and progression of asthma. The bronchial epithelial barrier consists of tight junctions, adherens junctions, desmosomes, hemidesmosomes and gap junctions, all of which are potentially implicated in asthma pathophysiology. In tight junctions, claudins, occludens, ZO-1 and $\beta$-catenin expression have been shown to be decreased by asthma allergens, resulting tight junction disruption. Similarly, E-cadherin and $\alpha$-catenin levels have also been reported to be dysregulated in response to asthma allergens, resulting in alterations in adherens junction structure. Asthma allergens also alter desmosome and hemidesmosome structure; however, no reports have shown that desmosome or hemidesmosome junction protein expression is altered in response to asthma allergens. Finally, in gap junctions, connexin 37 mRNA and protein were found to be decreased in the ovalbumin (OVA) induced allergic model. In summary, the regulation of bronchial junction protein expression and structure is an important and presently understudied component of asthma pathophysiology. We believe that further investigation in this area has the potential to aid in the development of novel asthma treatments.
\end{abstract}

Keywords: Bronchial epithelial; Junctional protein; Asthma; Barrier regulation

\section{Bronchial Epithelial Junction Structure}

Asthma is a complex inflammatory disorder that is characterized by multiple abnormalities in airway structure including epithelial fragility, bronchial structure remodeling, increased angiogenesis, goblet cell hyperplasia, and thickening of the lamina reticularis. Epithelial fragility enables tissue damaging agents to penetrate the airway wall, thus facilitating damage via toxic, immune and inflammatory responses [1]. The specific mechanisms of the epithelial changes underlying this fragility are an important component of asthma pathophysiology, but are presently incompletely understood. The bronchial epithelial barrier consists of tight junctions, adherens junctions, desmosomes, gap junctions and hemidesmosomes, which are each important to barrier integrity. Tight junctions (TJ) are essential for restricting fluid transport and maintaining the barrier properties of epithelial sheets [1]. Adherens junctions (AJ) form an adhesion belt around bronchial epithelial cells and are a critical link to cytoskeletal actin filaments. Desmosomes form cell-cell contacts and are linked to intermediate filaments. Gap junctions are composed of connexon channels that directly connect the cytoplasm of cells and allow the passage of small water-soluble ions and molecules. Hemidesmosomes resemble half of a desmosome and form cell-ECM junctions that serve to link the epithelial cells to the supporting basal lamina [2]. This review summarizes the current state of knowledge regarding changes to the bronchial epithelial junction in asthma with an emphasis on the mechanisms of structural changes and potential therapeutic implications.

\section{Epithelial Junction Structure Changes Induced by} Asthma

\section{The effect of asthma on tight junction structure}

Tight junctions (TJ) are located closest to the apical surface of epithelial cells and thus are the first junctions facing challenge from asthmatic allergens such as pollen or particulate matter (PM). Tight junction proteins include claudins, occludins, JAM1, zonaoccludens (ZOs), catenins, cingulin and F-actin [1]. Quantitative, structural, and biochemical analyses of tight junction dynamics following exposure of epithelial cells to house dust mite allergen Der $\mathrm{p} 1$ showed that Der $\mathrm{p} 1$ causes a time-dependent cleavage of TJs, a reduction in their content of the protein $\mathrm{ZO}-1$ and an increase in epithelial permeability, measured by mannitol clearance [3]. Immunoblotting showed that disruption of TJ morphology was associated with cleavage of ZO- 1 and occludin, and that cells recovered from allergen exposure through de novo synthesis of occludin. These results suggest that Der $\mathrm{p}$ 1contributes to the allergic responses seen in asthma by damaging tight junction proteins and thus disrupting epithelial barrier integrity [3].

In another study, asthma allergen Pen ch 13, a serine protease from $P$. chrysogenum, was found to cleave occludin in the human bronchial epithelial cell line 16HBE14o, thus disrupting tight junction structure [4]. This barrier disruption could facilitate passage of itself and other allergens to the submucosa, which is the location of the antigen presenting cells of the bronchial epithelium. Additionally, Pen ch 13 was shown to increase production of prostaglandin-E2 (PGE2), interleukin-8 (IL-8), and transforming growth factor- $\beta 1$ (TGF- $\beta 1$ ) by A549 cells, 16HBE14o cells, and primary cultures of human bronchial epithelial cells (HBEpCs) [4]. Furthermore it was found that Pen ch 13 protease activity is necessary for the induction of expression of PGE2, IL-8, TGF- $\beta 1$, and cyclooxygenase-2 (COX-2), suggesting that Pen ch 13 cleavage of occludin and subsequent barrier penetration facilitates sensitization and leads to the production of cytokines that are key to induction of a Th2 response (PGE2) and the recruitment of inflammatory cells to the airways (IL-8) [4].

*Corresponding author: Weiguo Chen, Section of Pulmonary, Critical Care Sleep and Allergy, University of Illinois at Chicago, COMRB 3160, MC 719, 909 S Wolcott Ave., Chicago IL 60612, USA, Tel: (312) 355-5931; Fax: (312) 996-4665 E-mail:weiguo@uic.edu

Received February 21, 2013; Accepted March 27, 2013; Published April 02, 2013

Citation: Xiao H, Rizzo AN, Siegler J, Chen W (2013) The Importance of Bronchia Epithelial Junction Integrity in Asthma. J Aller Ther S11: 003. doi:10.4172/21556121.S11-003

Copyright: (c) 2013 Xiao H, et al. This is an open-access article distributed unde the terms of the Creative Commons Attribution License, which permits unrestricted use, distribution, and reproduction in any medium, provided the original author and source are credited. 
In a recent study on epithelial barrier function in asthma, synthetic double stranded RNA polyinosinic:polycytidylic acid (poly I:C) was used to study the effects of respiratory viral infection, a common cause of asthma exacerbations, on epithelial junctional integrity [5]. Human bronchial epithelial cells and primary airway epithelial cells were grown on transwells, treated with poly I:C and assessed for barrier function by transepithelial resistance (TER) and paracellular flux of fluorescent markers. It was found that poly I:C decreased barrier integrity with a coincident reduction in junction localization of $\mathrm{ZO}-1$, occludin, E-cadherin, and $\beta$-catenin and disorganization of junction-associated actin filaments [5]. This critical finding suggests that viral infections may contribute to asthma exacerbations by decreasing the integrity of the epithelial barrier, thus allowing allergens to reach the submucosa to facilitate allergen sensitization.

A recent study of bronchial biopsies and primary bronchial epithelial cell (BEC) cultures from asthma patients found that tight junction structure and function was substantially altered. Immunostaining of bronchial biopsies and BEC cultures revealed that asthma subjects display a patchy disruption of TJs compared with normal controls. Furthermore, cultures from asthmatic donors were more sensitive to barrier disruption by cigarette smoke extract as measured by TER [6] These studies indicate that in both human samples and in vitro models bronchial epithelial barrier function is impaired in asthma and may facilitate the passage of allergens and other agents into the bronchial submucosa, leading to immune activation [6].

Additionally, another recent study found that siRNA directed against the epithelial tight junction protein claudin-1 affects airway smooth muscle (ASM) cell proliferation. This suggests that tight junction proteins may also play a role in airway remodeling in asthmatic patients through regulation of ASM cell proliferation, angiogenesis, and inflammation [7]. Additional evidence that tight junctions are critically important to asthma pathophysiology comes from recent genomic studies of tight junction proteins. A polymorphism in the $\beta$-catenin promoter was found to affect $\beta$-catenin expression efficiency and outcomes in asthmatic patients [8]. Similarly, an $\alpha$-catenin SNP was found that is associated with the severity of occupational asthma [9]. Collectively, the described research demonstrates that epithelial tight junctions are disrupted in asthma and that this may contribute to immune sensitization to allergens.

\section{The effect of asthma on adherens junction structure}

Below the tight junctions of bronchial epithelial cells, E-Cadherins connect to one another to form adherens junctions (AJ), which create an adhesion belt around the epithelial cells. AJs are associated with actin filaments by a complex that includes E-cadherin, plakoglobulin, p120, $\beta$-catenin and other proteins. A recent study comparing the AJ proteins E-cadherin, $\alpha$-catenin, and $\beta$-catenin in bronchial biopsies of atopic asthma patients and healthy controls showed that $\alpha$-catenin expression was significant lower in the epithelium of asthmatic patients and correlated inversely with the number of eosinophils within the epithelium [10]. Additionally, epithelial expression of ZO-1 and E-cadherin were significantly lower in asthmatic subjects than in nonasthmatic subjects. These results suggest that lower epithelial $\alpha$-catenin, E-cadherin, and ZO-1 expression in patients with atopic asthma increases in flux of eosinophils into the epithelium due to disruption of the airway epithelial barrier [10]. Thus, asthma allergens damage the epithelial barrier, at least in part, by regulating AJs and their associated actin filaments. Additionally, airway epithelium structure studies have shown that E-cadherin contributes to the structure and immunological functions of the airway epithelium through the regulation of epithelial junctions, proliferation, differentiation, and production of growth factors and pro-inflammatory mediators that can modulate the immune response [11].

\section{The effect of asthma on desmosome structure}

Desmosomes, which are located at the basolateral surface of epithelial cell junctions, play an important role in maintaining airway epithelial junction integrity. Desmosomes are composed of desmoplakin, keratin, and adherens proteins such as desmoglein, desmoculin, and others. By linking with intermediate filaments, desmosomes may regulate epithelial cytoskeletal rearrangement and responses to allergen challenge in asthma. Studies of epithelial shedding in the bronchial epithelium indicate that the bronchial epithelium is a truly stratified structure, that the superficial columnar cells depend on the underlying basal cells for anchorage and that desmosomes are the major structural proteins responsible for adhesion at the plane of cleavage between columnar cells and basal cells [12]. More recent studies utilized transmission electron microscopy to measure epithelial shedding and desmosome length in response to cytokine challenge, with or without co-administered corticosteroids. These studies revealed decreased desmosome length in the presence of cytokines (TNF-a and INF- $\gamma$ ) with an attenuation of this effect when corticosteroids were given [13]. This disruption of desmosome structure potentially facilitates inflammatory cell migration into lung tissue. Additionally, studies investigating epithelial damage in asthma at the ultra-structural level showed a 30-40\% decrease in desmosome length in both the basal and columnar cells. Reduced desmosomal contact may therefore be an important factor in the epithelial shedding observed in patients with asthma [14]. Additionally, ultrastructural investigations of epithelial damage in asthmatic and non-asthmatic nasal polyps showed that the length of columnar cell and basal cell desmosomes was reduced in allergic and asthmatic patients compared with those of control patients. Taken together, the evidence shows that desmosomes are structurally altered in asthmatic and allergic patients and suggests that epithelial shedding may play an important role in the pathophysiological processes of asthma and other allergic diseases [14,15].

\section{The effect of asthmatic allergens on gap junction structure}

Gap junctions are formed by six identical connexin units which together form a connexon, a hexagonal structure with a pore in the center. The diameter of the central pore is around $4 \mathrm{~nm}$, which allows for the passage of small water-soluble ions and molecules to pass between cells. Because gap junctions directly link the cytoplasm of adjacent cells, they have been implicated in intercellular signaling. In studies utilizing a murine model of ovalbumin- (OVA) induced allergic reaction it was found that expression of $\mathrm{Cx} 37 \mathrm{mRNA}$ and protein were decreased in OVA-induced allergic airway disease [16]. Assays showed that $\mathrm{Cx} 37$ was localized in epithelial layers around the bronchioles in control mice and that its expression was dramatically decreased in allergen-induced asthmatic lungs. Moreover, Cx37 protein level in lung tissue was negatively correlated with airway inflammation, airway responsiveness, and levels of Th2 cytokine in the lungs, suggesting that decreased Cx37 levels may be related to asthma pathophysiology [16].

\section{The effect of asthma on hemidesmosome structure}

A hemidesmosome resembles half of a desmosome and forms cell-ECM junctions. Hemidesmosomes in airway epithelial cells serve to attach them to the underlying basal lamina. Desmopenetrin is the adhesion protein in hemidesmosomes, compared to desmogleins in desmosomes. Hemidesmosomes combine with the integrin a6- $\beta 4-$ 
complex to anchor basal and columnar cells to the underlying basement membrane [12]. The fact that epithelial cells become vulnerable and more cells are lost in clusters in asthma patients suggests that these hemidesmosome connections are altered by allergens [12]. These findings were validated in a study that examined bronchoalveolar lavage (BAL) samples from asthmatic and control subjects. The BAL of atopic asthma patients showed a higher ratio of columnar cells to basal cells in compared to controls indicating that shedding of epithelial cells occurred primarily due to cleavage between the suprabasal and the basal cell layers, which are usually anchored by hemidesmosomes [17].

\section{Junction Proteins were Therapeutic Targets in Asthmatic Patients}

\section{Tight junction proteins as therapeutic targets}

As the asthma-induced changes in airway epithelial junctions are becoming better characterized, scientists are now attempting to develop novel asthma therapeutics to target these changes. Specifically, LipoxinA (4) (LXA(4)) has been shown to increase expression of ZO-1, claudin-1, and occludinin confluent $16 \mathrm{HBE} 14 \mathrm{o}$ cells with coincident increases in cell junction integrity [18]. Additionally, respiratory syncytial virus (RSV) budding studies in upper airway nasal epithelial cells (HNECs) showed that RSV infection caused an increase in proinflammatory cytokines IL-8 and TNF- $\alpha$, which in turn up-regulated claudin-4 and occluding expression. The replication and budding of RSV and the epithelial cell responses in HNECs were found to be regulated via a protein kinase $C \delta /$ hypoxia-inducible factor-1 $\alpha /$ nuclear factor- $\kappa \mathrm{B}$ pathway indicating that control of this pathway in HNECs may be useful not only for preventing replication and budding of RSV, but also in treating RSV-induced respiratory pathology [19].

Another study looked at the tight junction permeability of ovalbumin-sensitized trachea in guinea pigs and found geniposide significantly decreased permeability. Additionally, they noted that the removal of extracellular $\mathrm{Ca}^{2+}$ abolished this beneficial effect indicating that geniposide has inhibitory effects on ovalbumin-induced junction permeability through a calcium dependent mechanism [20]. Finally, glucocorticoids (GCs), display strong anti-asthma effects accompanying the enhancement of human airway epithelial cell junction integrity. Furthermore, silencing epidermal growth factor receptor (EGFR) by siRNA blunted the effects of GCs on barrier integrity suggesting that inhalation GCs improve epithelial barrier integrity, which may contribute to their therapeutic effects in asthma [21].

\section{Adherens junction proteins as therapeutic targets}

Due to their implications in asthma pathophysiology, adherens junction proteins have also been considered for the development of novel asthma therapeutics. Specifically, a curcumin derivative, CNB001, has shown a strong anti-inflammatory effect in a NHBE human bronchial epithelial cell asthma model by attenuating the decrease in E-cadherin expression [22]. Additionally, a combination of TGF- $\beta 1$ and TNF- $\alpha$, key markers of airway remodeling, induced an increase in vimentin mRNA expression in NHBE cells [22]. Another study looked at the effects of TGF- $\beta 1$ on beas $2 b$ (normal bronchial epithelial cells) and primary human bronchial epithelial cultures and found that TGF- $\beta 1$ reduced expression levels of the adherens junction protein E-cadherin. Since asthma is associated with an increase in TGF- $\beta 1$ expression therapeutics targeting this pathway may aid in epithelial barrier function at the level of adherens junction protein expression and have the potential to be useful in asthma [23].
Another study on E-cadherin in asthma found that E-cadherin is partly cleaved and released into the lumen as part of the late airway response (LAR) that occurs 6 hours after ovalbumin challenge in guinea pigs [24]. This study indicates that antigen challenge causes an opening of adherens junctions as well as increased airway permeability in LAR [24]. In addition, E-cadherin (CDH1) gene polymorphisms have a close relationship with airway remodeling, inflammation, and the decline in forced expiratory volume in $1 \mathrm{~s}(\mathrm{FEV}(1))$ in asthma patients, as well as with their response to corticosteroid treatment [25].

\section{Gap junction proteins as therapeutic targets}

Inhibition of gap junction intercellular communication and silencing of connexin have been shown to modulate the course of asthma development. Connexin-based channels i) coordinate ciliary beating and fluid transport to promote clearance of particulates, ii) regulate secretion of pulmonary surfactant in response to deep inhalation by interconnecting type 1 and type 2 alveolar epithelial cells, and iii) are key mediators of pro- and anti-inflammatory signaling by the pulmonary endothelium that modulate leukocyte recruitment from the circulation. Thus, connexin-based channels play several central roles in promoting a regulated inflammatory response and facilitating lung repair, which enables the pulmonary epithelium and vasculature to behave as an integrated system. This makes the regulation of connexinbased channels a potential target for novel asthma therapeutic interventions [26].

\section{Desmosome and Hemidesmosome proteins as therapeutic targets}

There are no reports regarding medications targeting desmosome or hemidesmosome proteins for asthma treatment.

\section{Perspective on Studying Bronchial Junction Proteins in Asthma}

As we reviewed in this paper, there is a long way to go to completely elucidate the mechanisms of bronchial junction damage induced by asthma. However, it is clear that disruption of the physical barrier of the bronchial epithelium is implicated in asthma and may facilitate the passage of allergens to the underlying submucosa, thus contributing to the development of immune responses. The multitude of immune responses and the downstream signaling pathways that are involved indicate the complexity of the epithelial junction changes induced by asthma. Another area of increasing interest and added complexity is the contribution of SNPs in junctional proteins and barrier regulatory proteins to asthma severity. As noted above, SNPs in $\beta$ catenin and a catenin that are associated with increased asthma severity have been identified [8,9]. Additionally, one recent study showed that intronic SNPs in myosin light-chain kinase (MYLK), the gene that encodes critical the cytoskeletal effector protein MLCK, are related to severity of acute lung injury and asthma [27]. Thus, cytoskeletal barrier regulatory proteins in addition to the barrier proteins themselves must be studied in order to get a full understanding of how the epithelial barrier contributes to asthma pathophysiology.

\section{Acknowledgement}

This study was supported by National Natural Scientific Foundation of China (NSFC-81270133). The funders had no role in study design, data collection and analysis, decision to publish, or preparation of the manuscript.

\section{References}

1. Holgate ST (2008) The airway epithelium is central to the pathogenesis of asthma. Allergol Int 57: 1-10. 
Citation: Xiao H, Rizzo AN, Siegler J, Chen W (2013) The Importance of Bronchial Epithelial Junction Integrity in Asthma. J Aller Ther S11: 003. doi:10.4172/2155-6121.S11-003

2. Bérubé K, Prytherch Z, Job C, Hughes T (2010) Human primary bronchial lung cell constructs: the new respiratory models. Toxicology 278: 311-318.

3. Wan H, Winton HL, Soeller C, Gruenert DC, Thompson PJ, et al. (2000) Quantitative structural and biochemical analyses of tight junction dynamics following exposure of epithelial cells to house dust mite allergen Der p 1. Clin Exp Allergy 30: 685-698.

4. Tai HY, Tam MF, Chou H, Peng HJ, Su SN, et al. (2006) Pen ch 13 allergen induces secretion of mediators and degradation of occludin protein of human lung epithelial cells. Allergy 61: 382-388.

5. Rezaee F, Meednu N, Emo JA, Saatian B, Chapman TJ, et al. (2011) Polyinosinic:polycytidylic acid induces protein kinase D-dependent disassembly of apical junctions and barrier dysfunction in airway epithelial cells. J Allergy Clin Immunol 128: 1216-1224.

6. Xiao C, Puddicombe SM, Field S, Haywood J, Broughton-Head V, et al. (2011) Defective epithelial barrier function in asthma. J Allergy Clin Immunol 128: 549556

7. Fujita $\mathrm{H}$, Chalubinski M, Rhyner C, Indermitte $\mathrm{P}$, Meyer $\mathrm{N}$, et al. (2011) Claudin-1 expression in airway smooth muscle exacerbates airway remodeling in asthmatic subjects. J Allergy Clin Immunol 127: 1612-1621.

8. Bae S, Lee H, Choi BW, Lee HK, Chung SI, et al. (2012) Beta-catenin promoter polymorphism is associated with asthma risk in Korean subjects. Clin Biochem 45: 1187-1191.

9. Bernstein DI, Kashon M, Lummus ZL, Johnson VJ, Fluharty K, et al. (2013) CTNNA3 (Ît-catenin) gene variants are associated with diisocyanate asthma: a replication study in a caucasian worker population. Toxicol Sci 131: 242-246.

10. de Boer WI, Sharma HS, Baelemans SM, Hoogsteden HC, Lambrecht BN, et al. (2008) Altered expression of epithelial junctional proteins in atopic asthma: possible role in inflammation. Can J Physiol Pharmacol 86: 105-112.

11. Nawijn MC, Hackett TL, Postma DS, van Oosterhout AJ, Heijink IH (2011) E-cadherin: gatekeeper of airway mucosa and allergic sensitization. Trends Immunol 32: 248-255.

12. Roche WR, Montefort S, Baker J, Holgate ST (1993) Cell adhesion molecules and the bronchial epithelium. Am Rev Respir Dis 148: S79-82.

13. Andersson K, Shebani EB, Makeeva N, Roomans GM, Servetnyk Z (2010) Corticosteroids and montelukast: effects on airway epithelial and human umbilical vein endothelial cells. Lung 188: 209-216.

14. Shahana S, Jaunmuktane Z, Asplund MS, Roomans GM (2006) Ultrastructural investigation of epithelial damage in asthmatic and non-asthmatic nasal polyps. Respir Med 100: 2018-2028.
15. Shahana S, Björnsson E, Lúdvíksdóttir D, Janson C, Nettelbladt O, et al. (2005) Ultrastructure of bronchial biopsies from patients with allergic and non-allergic asthma. Respir Med 99: 429-443.

16. Park SJ, Lee KS, Kim SR, Min KH, Lee KY, et al. (2007) Change of connexin 37 in allergen-induced airway inflammation. Exp Mol Med 39: 629-640.

17. Montefort S, Herbert CA, Robinson C, Holgate ST (1992) The bronchia epithelium as a target for inflammatory attack in asthma. Clin Exp Allergy 22 511-520.

18. Grumbach Y, Quynh NV, Chiron R, Urbach V (2009) LXA4 stimulates ZO-1 expression and transepithelial electrical resistance in human airway epithelia (16HBE14o-) cells. Am J Physiol Lung Cell Mol Physiol 296: L101-108.

19. Masaki T, Kojima T, Okabayashi T, Ogasawara N, Ohkuni T, et al. (2011) A nuclear factor-kB signaling pathway via protein kinase $\mathrm{C} d$ regulates replication of respiratory syncytial virus in polarized normal human nasal epithelial cells. Mol Biol Cell 22: 2144-2156.

20. Liaw J, Chao YC (2001) Effect of in vitro and in vivo aerosolized treatment with geniposide on tracheal permeability in ovalbumin-induced guinea pigs. Eur $J$ Pharmacol 433: 115-121.

21. SekiyamaA, Gon Y, Terakado M, Takeshita I, Kozu Y, et al. (2012) Glucocorticoids enhance airway epithelial barrier integrity. Int Immunopharmacol 12: 350-357.

22. Narumoto O, Matsuo Y, Sakaguchi M, Shoji S, Yamashita N, et al. (2012) Suppressive effects of a pyrazole derivative of curcumin on airway inflammation and remodeling. Exp Mol Pathol 93: 18-25.

23. Doerner AM, Zuraw BL (2009) TGF-beta1 induced epithelial to mesenchyma transition (EMT) in human bronchial epithelial cells is enhanced by IL-1beta but not abrogated by corticosteroids. Respir Res 10: 100.

24. Goto Y, Uchida Y, Nomura A, Sakamoto T, Ishii Y, et al. (2000) Dislocation of E-cadherin in the airway epithelium during an antigen-induced asthmatic response. Am J Respir Cell Mol Biol 23: 712-718.

25. lerodiakonou D, Postma DS, Koppelman GH, Boezen HM, Gerritsen J, et al. (2011) E-cadherin gene polymorphisms in asthma patients using inhaled corticosteroids. Eur Respir J 38: 1044-1052.

26. Losa D, Chanson M, Crespin S (2011) Connexins as therapeutic targets in lung disease. Expert Opin Ther Targets 15: 989-1002.

27. Han YJ, Ma SF, Wade MS, Flores C, Garcia JG (2012) An intronic MYLK variant associated with inflammatory lung disease regulates promoter activity of the smooth muscle myosin light chain kinase isoform. J Mol Med (Berl) 90: 299308

This article was originally published in a special issue, Asthma handled by Editor. Dr. Manar A Nader, Mansoura University, Egypt 